\title{
Potencial agroclimático para a Alpinia purpurata, no Estado de Pernambuco ${ }^{1}$
}

\author{
Sérgio R. R. Medeiros², Geber B. A. Moura ${ }^{3}$, Pedro R. Giongo ${ }^{2}$ \& Ana P. N. Silva ${ }^{2}$
}

\begin{abstract}
RESUMO
Objetivou-se realizar o zoneamento agroclimático do Estado de Pernambuco, visando dar subsídios à seleção de locais para a implantação da flor tropical Alpinia purpurata. Dados climáticos de 123 localidades foram utilizados, dos quais 69 com uma série acima de 30 anos de observações e 54 abaixo de 30 anos de observações obtendo-se assim, melhor representatividade de dados no Estado. Dos dados climáticos de temperaturas médias do ar e precipitação pluviométrica, calcularam-se os balanços hídricos climatológicos. Escolheram-se os municípios de Igarassu, Primavera, Goiana e São Lourenço da Mata para serem representativos climatológicos, por serem produtores da espécie em Pernambuco. De posse dos dados dos balanços hídricos e das temperaturas mínima e máxima do ar, se estimaram os índices de limite térmico e hídrico em relação às exigências da espécie nos municípios representativos e só então estes índices foram extrapolados para todo o Estado. De acordo com o estudo, Pernambuco apresentou 6 localidades com aptidão plena, 16 com aptidão regular e 101 com inaptidão à espécie.
\end{abstract}

Palavras-chave: zoneamento, flor tropical, índices térmico e hídrico

\section{Agroclimatic potencial for Alpinia purpurata in the State of Pernambuco}

\begin{abstract}
This study aimed at accomplishing the agroclimatic zoning of Pernambuco State, in order to provide subsidies for the selection of areas for implantation of Alpinia purpurata tropical flower. The climatic data of 123 localities (69 with a period above 30 years and 54 below 30 years) were used, thus getting a better representation of the data in the state. From climatic data of the average temperatures of air and rainfall, the climatologic water balance has been calculated. The cities of Igarassú, Primavera, Goiana and São Lourenço da Mata were chosen to be climatic representatives as they are the producers of the species in Pernambuco. By having the data of the water balance and the minimum and maximum air temperatures, the indexes of thermal and water limit in relation to the requirements of the species in the representative cities has been estimated, and only after this the indexes were extrapolated for the whole State. In accordance with this study, Pernambuco presented 6 localities with full suitability, 16 with regular suitability and 101 unsuitable for the cultivation of species.
\end{abstract}

Key words: zoning, tropical flower, thermal and hydric indexes

\footnotetext{
Parte da Dissertação de Mestrado do primeiro autor, apresentada à UFRPE.

DTR/UFRPE. Rua Dom Manoel de Medeiros s/n, Dois Irmãos, CEP 52171-900, Recife, PE. E-mail: medeirossoft@gmail.com

DEPA/UFRPE. Fone: (81) 3320-6246. E-mail: geber@depa.ufrpe.br
} 


\section{INTRODUÇÃO}

No planejamento e programação das atividades rurais, uma ferramenta imprescindível é a avaliação do potencial agroclimático das áreas, conforme a necessidade das culturas. O zoneamento agroclimático auxilia na tomada de decisão dos produtores rurais, que visam a um aproveitamento melhor dos recursos naturais da propriedade, além de orientar ações de prefeituras que pretendam incentivar a agropecuária em seu município (Assad, 1998).

O mercado mundial de flores e plantas ornamentais gera um fluxo anual no comércio internacional, da ordem de 6,6 bilhões de dólares; hoje, concentrado em países como Holanda, Colômbia, Itália, Dinamarca, Bélgica, Quênia, Zimbábue, Costa Rica, Equador, Austrália, Malásia, Tailândia, Israel e EUA, a participação brasileira é de apenas 0,3\% no fluxo internacional (Junqueira \& Peetz, 2002).

No Brasil, as flores tropicais são apontadas como de grande potencial estratégico de crescimento no mercado internacional, situando-se Pernambuco como um dos principais exportadores, refletindo notável especialização deste setor. Ante tais perspectivas, o Estado de Pernambuco se vem organizando com o intuito de se tornar mais competitivo no setor da floricultura e um expoente na exportação de flores (Junqueira \& Peetz, 2002).

Em Pernambuco, o desenvolvimento da floricultura tropical foi significativo nos últimos anos, sendo considerado, hoje, um dos estados que mais investiram no setor, não apenas em produção mas também em pesquisas e exportação. Atualmente, a produção de flores tropicais é conduzida por pequenos produtores que detêm áreas em média de 5 ha (Ferreira et al., 2002). São cultivadas, no estado, várias espécies de flores de corte das famílias Heliconiaceae, Zingiberaceae, Costaceae, Araceae, além de espécies com folhagens de corte.

A Alpinia purpurata é uma planta tropical pertencente à família Zingiberaceae. Cultivada há muito tempo como planta ornamental em paisagismo, seu porte oscila de 1,5 até 4,0 m de altura; suas inflorescências são terminais, envoltas em folhas apicais, que podem chegar de 15 a $30 \mathrm{~cm}$ de comprimento e atingir tamanhos maiores com a idade mais avançada.

Recentemente, seu uso potencial como flor de corte, devido à durabilidade e exuberância de suas inflorescências aliadas ao florescimento contínuo durante o ano todo, foi reconhecido e existem, hoje, produções comerciais significativas no Havaí, América do Sul e Central (Lamas, 2002).

O cultivo da alpínia se desenvolve bem a meia sombra (em especial as de coloração rosácea); sob um sombreamento de 20 a $45 \%$, as plantas apresentam bom crescimento vegetativo e florescimento adequado; a necessidade luminosa oscila de 50.000 a 75.000 lux $(0,000007321$ a $0,000010249 \mathrm{~W} \mathrm{~cm}^{-2}$ ).

A faixa de temperatura de cultivo adequada está situada entre 22 e $35^{\circ} \mathrm{C}$, com temperatura máxima noturna de $27^{\circ} \mathrm{C}$ e mínima de $18{ }^{\circ} \mathrm{C}$; a temperatura ótima para a produção está entre 24 e $30^{\circ} \mathrm{C}$, enquanto a umidade relativa do ar deve oscilar entre 60 a $80 \%$. (Lamas, 2002).
Em Pernambuco, praticamente se dispõe apenas de postos pluviométricos, razoavelmente distribuídos; o número de estações meteorológicas é relativamente pequeno, impossibilitando estudos que envolvam a distribuição espacial da maior parte das variáveis climáticas (umidade, vento, radiação, evaporação, orvalho, etc.)

A precipitação pluvial em regiões tropicais é bastante variável no tempo, espaço, duração e quantidade, enquanto a temperatura do ar apresenta baixa variabilidade, portanto, facilmente modelada em função das coordenadas geográficas (Cavalcanti et al., 2006).

Propôs-se, no presente trabalho, realizar o zoneamento agroclimático para o Estado de Pernambuco, no tocante à classificação climática regional para a Alpinia purpurata, com base nas exigências térmicas e hídricas da cultura.

\section{MATERIAL E MÉTODOS}

O Estado de Pernambuco possui uma área de 98.937,8 km² e está localizado entre as Latitudes de $7^{\circ} 20^{\prime}$ a $9^{\circ} 29^{\prime}$ S e Longitudes $34^{\circ} 30^{\prime}$ a $41^{\circ} 21^{\prime}$ W, no Nordeste brasileiro.

Utilizam-se, para a realização do zoneamento da Alpinia purpurata, dados climáticos de 123 postos, do quais 69 postos com uma série acima de 30 anos de observações e 54 postos em uma série abaixo de 30 anos de observações para melhor representatividade de dados para o Estado. Os dados climáticos mencionados são as médias anuais de temperatura e precipitação (SUDENE, 1990), disponíveis na página virtual da Unidade Acadêmica de Ciências Atmosféricas (UACA) da Universidade Federal de Campina Grande UFCG.

A baixa concentração espacial de locais com registros termométricos restringe drasticamente a disponibilidade de dados de temperatura, quando comparados aos de precipitação; para contornar este problema, estimou-se a média mensal de temperatura do ar nos locais em que apenas se dispunham de dados de chuva; nessa estimativa, os valores médios mensais disponíveis no Estado de Pernambuco e nos estados vizinhos foram utilizados, considerando-se tanto as normais climatológicas, fornecidas pelo Instituto Nacional de Meteorologia (INMET), como outras fontes (SUDENE, 1963; DRA, 1967; Ellis \& Valença, 1982); além do modelo de regressão múltipla quadrática, segundo Cavalcanti \& Silva (1994). A estimativa da temperatura é função da latitude $(\varphi)$, da longitude $(\lambda)$ e da altitude (h) como variáveis independentes:

$$
\begin{aligned}
\mathrm{T}= & \mathrm{A}_{0}+\mathrm{A}_{1} \lambda+\mathrm{A}_{2} \varphi+\mathrm{A}_{3} \mathrm{~h}+\mathrm{A}_{4} \lambda^{2}+\mathrm{A}_{5} \varphi^{2}+\mathrm{A}_{6} \mathrm{~h}^{2}+ \\
& \mathrm{A}_{7} \lambda \varphi+\mathrm{A}_{8} \lambda \mathrm{h}+\mathrm{A}_{9} \varphi \lambda
\end{aligned}
$$

Obtiveram-se os coeficientes $A_{0}, A_{1}, \ldots, A_{9}$ pelo método dos mínimos quadrados, o que consiste na solução de vários sistemas de 10 equações a 10 incógnitas, devendo corresponder a cada mês e a cada sub-região.

Segundo Hagiladi et al. (1997) em geral as Zingiberáceas crescem menos a baixas temperaturas e dias curtos (menos de 12 horas); trata-se de plantas perenes tropicais e subtropicais 
nativas de áreas com pouca variação de temperatura e de dias longos durante o ano (Zhang et al., 1995).

Em Pernambuco, por se encontrar em baixas latitudes, não há uma variação expressiva no fotoperíodo que permanece em torno de 12 h, praticamente durante todo o ano; logo, não é fator limitante e, sendo assim, o zoneamento apenas será de natureza térmica e hídrica.

De posse dos dados da temperatura média e da precipitação média dos postos, calcularam-se os balanços hídricos, de acordo com Thornthwaite \& Mather (1955) para uma capacidade de retenção de água no solo de 100 mm (CAD), normalmente utilizado como média climatológica para a região.

A amplitude térmica exigida para o cultivo da Alpinia purpurata se encontra entre 22 a $35^{\circ} \mathrm{C}$. Pernambuco não apresenta temperatura média superior a $35^{\circ} \mathrm{C}$ mas apenas temperaturas abaixo de $22^{\circ} \mathrm{C}$ em algumas regiões, como observou Lamas (2002).

Na Figura 1 apresentam-se alguns municípios nos quais se destaca a produção da A.purpurata em Pernambuco: Igarassu, Paulista, Recife, Goiana, Camaragibe, Moreno, Cabo, São Lourenço da Mata, Ipojuca, Ribeirão, Primavera, Água Preta, Gravatá e Petrolina.

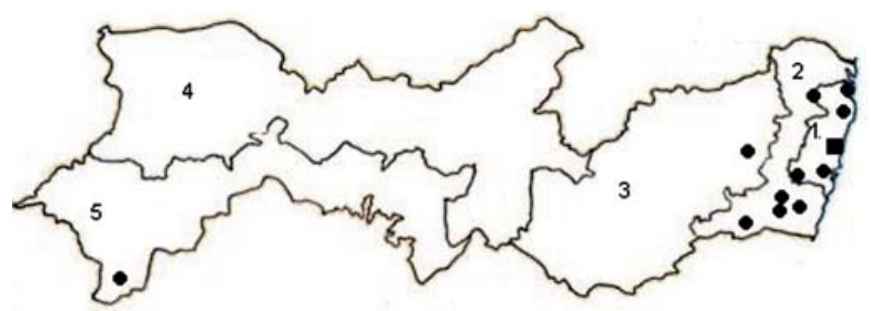

Figura 1. Mapa do Estado de Pernambuco com as Mesorregiões: 1 - Região Metropolitana; 2 - Zona da Mata; 3 - Agreste; 4 - Sertão, e 5 - Sertão de São Francisco

Foram adotados, em relação à temperatura, os valores térmicos segundo Lamas (2002), citados anteriormente, como faixa de aptidão agroclimática; para o déficit hídrico foram adotados quatro municípios como representativos da produção de $A$. purpurata em Pernambuco, a partir dos quais se estabeleceram os limites para o déficit hídrico; os quatros municípios escolhidos, foram: Igarassu, Goiana, São Lourenço da Mata e Primavera, haja vista que, dos municípios produtores já citados, há dados de pluviosidade e de temperatura média do ar.

Os municípios de Igarassu e Primavera apresentaram um déficit hídrico anual de aproximadamente $200 \mathrm{~mm}$, enquanto em São Lourenço da Mata e Goiana este déficit foi de aproximadamente $400 \mathrm{~mm}$ anuais (Tabela 1).

Com base nos dados desses municípios se estabeleceram os limites térmicos e hídricos extrapolando-os, em seguida, para todo o Estado encontrando-se assim, regiões de semelhança em relação aos limites já citados para a geração das cartas de temperatura média anual mínima e máxima, do déficit hídrico anual, da evapotranspiração potencial anual e do zoneamento agroclimático da espécie.
Tabela 1. Valores médios anuais de temperatura (mínima e máxima), déficit hídrico e evapotranspiração potencial dos postos representativos de Pernambuco

\begin{tabular}{lcccccc}
\hline Local & Long. & Lat. & $\begin{array}{c}\text { Temp. } \\
\text { mín. } \\
\left({ }^{\circ} \mathbf{C}\right)\end{array}$ & $\begin{array}{c}\text { Temp. } \\
\text { máx. } \\
{ }^{\left({ }^{\circ} \mathbf{C}\right)}\end{array}$ & $\begin{array}{c}\text { Déficit } \\
\text { hídrico } \\
(\mathbf{m m})\end{array}$ & $\begin{array}{c}\text { ETp } \\
(\mathbf{m m})\end{array}$ \\
Primavera & $-35,35$ & $-8,35$ & 22,6 & 26,0 & 164,3 & 1329,1 \\
Igarassu & $-34,90$ & $-7,83$ & 23,3 & 26,4 & 203,5 & 1399,6 \\
São Lourenço da Mata & $-35,05$ & $-8,00$ & 23,1 & 26,3 & 375,5 & 1376,5 \\
Matari/goiana & $-35,13$ & $-7,66$ & 23,2 & 26,3 & 382,2 & 1387,4 \\
\hline
\end{tabular}

\section{RESULTADOS E DISCUSSÃO}

De posse dos dados dos balanços hídricos dos postos analisados de Pernambuco, geraram-se as cartas de temperaturas médias mínima e máxima anuais, os déficits hídricos e a evapotranspiração potencial anual.

Na determinação dos índices-limite, dos fatores térmico e hídrico, Pernambuco apresentou temperaturas inferiores em médias anuais, variando de 19,2 a $28,1^{\circ} \mathrm{C}$; portanto, o déficit hídrico não pode ser o único parâmetro climático determinante para o zoneamento e, sim, uma relação algorítmica dos dois índices; desta forma, aparecem três faixas de condições climáticas em Pernambuco, em relação ao cultivo da Alpinia purpurata, ou seja:

- Zona apta: condições térmicas e hídricas satisfatórias, onde a temperatura é maior que $22^{\circ} \mathrm{C}$ e o déficit hídrico $\leq 200 \mathrm{~mm}$ por ano.

- Zona Restrita (moderadamente apta): condições térmicas satisfatórias e deficiências hídricas moderadas, isto é, temperatura superior a $22{ }^{\circ} \mathrm{C}$ e déficit hídrico compreendido entre 200 e 400 mm, cujo cultivo já necessita ser suplementado com irrigação, além de outras técnicas no manejo para o desenvolvimento da cultura.

- Zona inapta: condições térmicas e hídricas desfavoráveis, ou seja, a temperatura é inferior a $22^{\circ} \mathrm{C}$ e o déficit hídrico maior que $400 \mathrm{~mm}$ ou a temperatura maior que $22{ }^{\circ} \mathrm{C}$ e o déficit também superior a $400 \mathrm{~mm}$, chegando à inviabilidade do cultivo da flor a baixos custos ocorrendo, assim, a necessidade de técnicas mais sofisticadas e mão-de-obra especializada.

A Figura 2 apresenta a Carta do Zoneamento Agroclimático da Alpinia purpurata em Pernambuco.

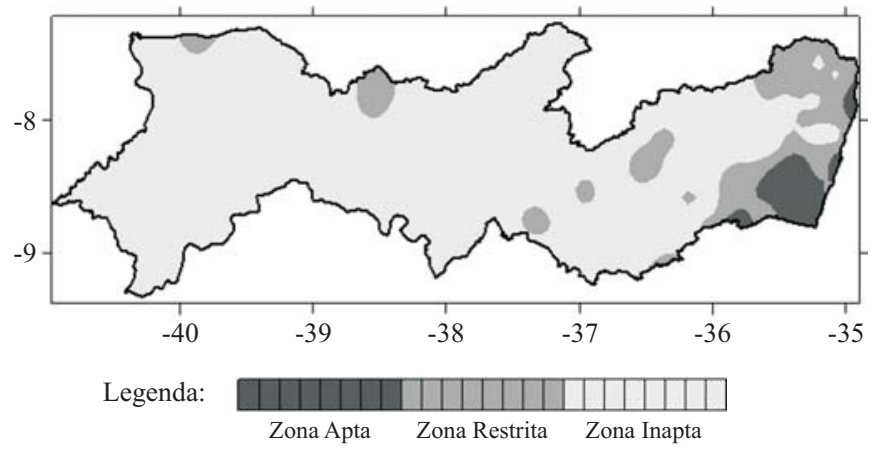

Figura 2. Carta do Zoneamento Agroclimático da Alpinia purpurata,em Pernambuco 
Segundo Varejão-Silva \& Cezar-Barros (2001) há incerteza quanto à posição geográfica do traçado das linhas que separam as diferentes classes de aptidão climática, em virtude da distribuição espacial dos postos pluviométricos no Estado de Pernambuco, que ainda está bem aquém do desejável, deixando algumas áreas sem nenhuma informação, dificultando o traçado das linhas, isto é, do limite das faixas de aptidão estimadas.

Cada faixa de aptidão deve ser considerada também como área de transição, em que as condições climáticas vão gradualmente mudando, quando se parte da porção central da faixa em direção às faixas vizinhas.

Foram analisadas as zonas agroclimáticas e descritos os intervalos de temperatura, déficit hídrico e evapotranspiração para cada zona agroclimática de Pernambuco para a Alpinia purpurata:

Na zona apta, a Figura 3A apresentou um intervalo da tem-

A.

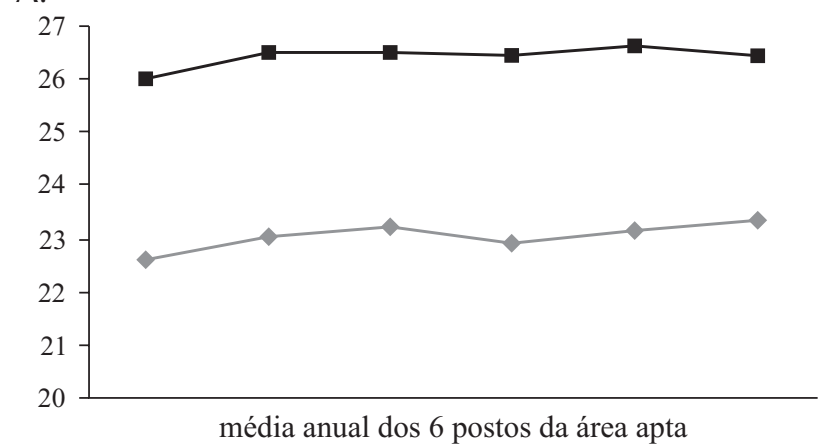

C.

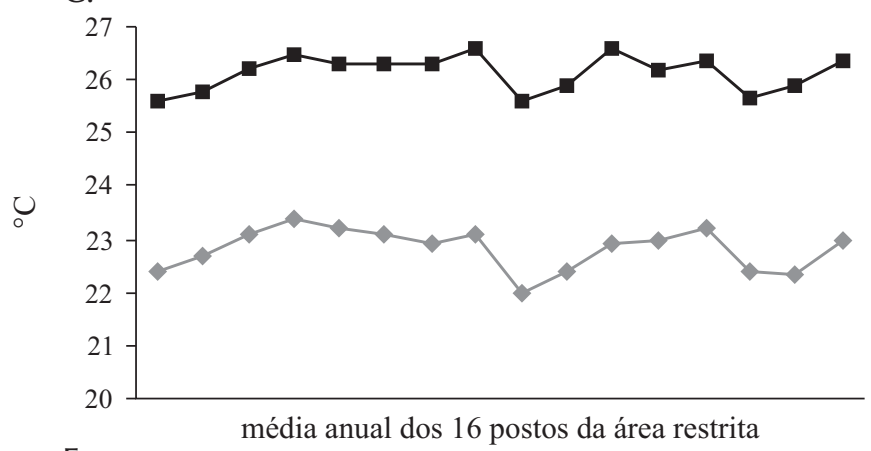

E.

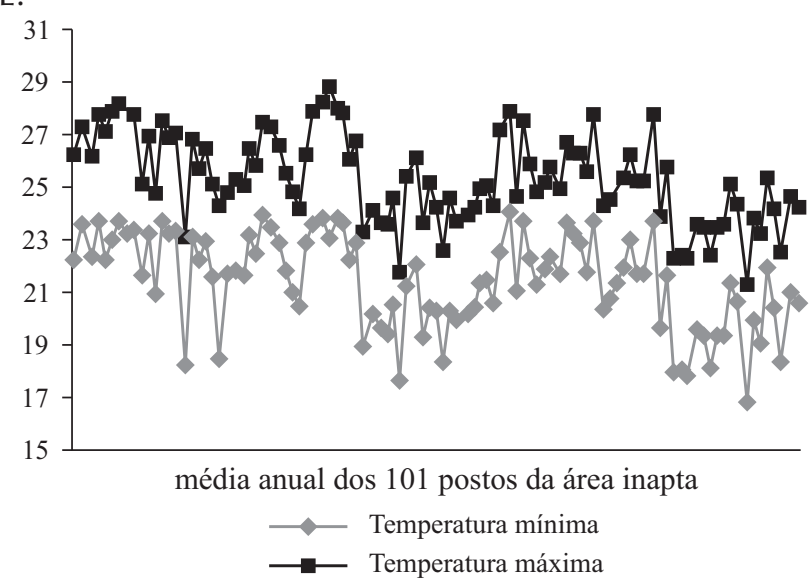

peratura média anual entre a mínima e máxima de 22,6 a $26,6^{\circ} \mathrm{C}$; a Figura 3B apontou a variação da evapotranspiração potencial anual entre 1.329 a 1.420 mm e o déficit hídrico anual compreendido no intervalo entre 155 a $200 \mathrm{~mm}$.

Na zona restrita, a Figura 3C indicou a temperatura média anual entre a mínima e máxima variando entre 22 a 26, $6^{\circ} \mathrm{C}$; a Figura 3D apresentou uma evapotranspiração potencial anual, que oscilou entre 1.227 a $1.414 \mathrm{~mm}$ e o déficit hídrico anual, entre 245 a $397 \mathrm{~mm}$.

Vê-se na Figura 3E da zona inapta, a variação da temperatura média anual entre a mínima e máxima em um intervalo de 18,6 a $28,7^{\circ} \mathrm{C}$; na Figura $3 \mathrm{~F}$ uma oscilação da evapotranspiração potencial anual entre 915 a $1.536 \mathrm{~mm}$, enquanto o déficit hídrico anual dentro do intervalo é de 94,8 a $1.132 \mathrm{~mm}$.

Embora a zona inapta apresentasse regiões com déficit hídrico menor que $200 \mathrm{~mm}$ (limite para a zona apta) ou
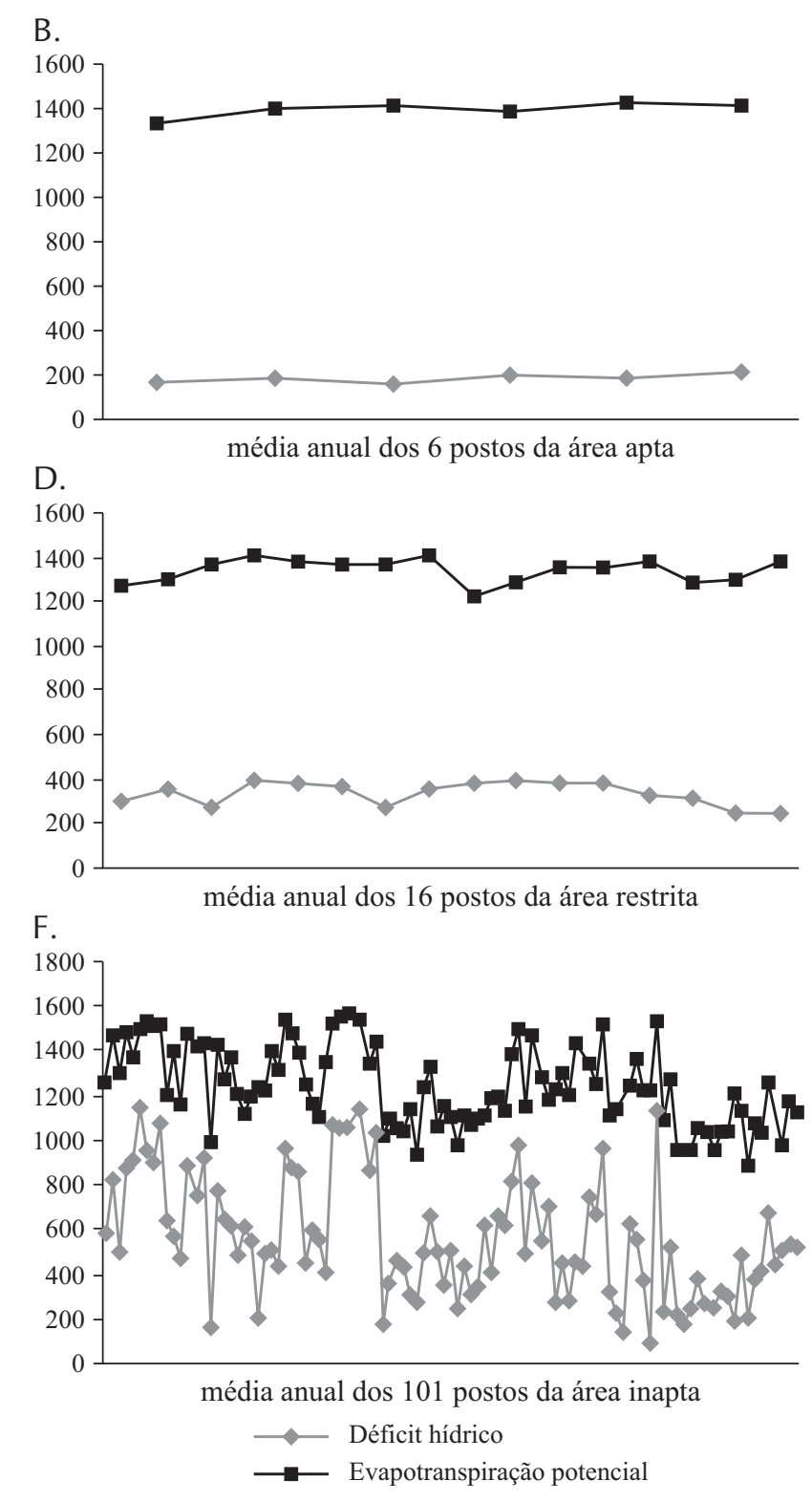

Figura 3. Descrição dos intervalos da temperatura média máxima e mínima (A, C, E), déficit hídrico e evapotranspiração potencial (B, D, F) para cada zona agroclimática de Pernambuco 
mesmo entre 200 a $400 \mathrm{~mm}$ (intervalo limite para a zona restrita), as temperaturas médias, associadas a esses déficits, são normalmente inferiores a $22{ }^{\circ} \mathrm{C}$ como, por exemplo, o município de Buíque, cuja temperatura variou entre $19^{\circ} \mathrm{C}$ (média mínima anual) a $23,2^{\circ} \mathrm{C}$ (média máxima anual) e apresentou um déficit hídrico acumulado de 176,7 mm.

Reis (1972), ao analisar a viabilidade climática para a cafeicultura em Pernambuco, ressalta que não só para o cafeeiro mas também em relação a qualquer cultura para a qual se venha tentar estabelecer índices-limite para o seu zoneamento em uma região como o Nordeste, deve-se ter em mente a baixa produtividade dos solos em comparação com a produtividade média dos solos do Sudeste.

Como já mencionado na metodologia, o zoneamento foi de natureza térmica (temperatura) e hídrica (déficit hídrico e evapotranspiração potencial) e não houve restrição quanto ao fotoperíodo, pois Pernambuco se localiza em latitudes baixas, entre $7^{\circ} 20^{\prime}$ a $9^{\circ} 29^{\prime}$, a variação do fotoperíodo é de $11 \mathrm{~h}$ e 56 min às 12 h e 65 min, praticamente não variando; portanto, dentro das $12 \mathrm{~h}$, não comprometendo o crescimento das Zingeberáceas, entre elas a A. purpurata.

\section{CONCLUSÕES}

1. O mapeamento das isotermas e do déficit hídrico climatológico permite delimitar as zonas com diferentes aptidões agroclimáticas para o cultivo da Alpinia purpurata, no Estado de Pernambuco.

2. A zona apta se concentrou em 6 localidades: Barreiros, Cabo, Gameleira, Igarassu, Primavera e Rio Formoso, distribuídas na Mesorregião Metropolitana do Recife e, sobretudo, na Mesorregião da Zona da Mata Pernambucana, na área sul, onde existe uma boa distribuição temporal de chuvas.

3. A zona restrita se restringiu a 16 localidades distribuídas na Mesorregião Metropolitana de Recife, na Mesorregião da Zona da Mata Pernambucana, na fronteira entre a Zona da Mata e o Agreste, surgindo algumas “ilhas” de zona restrita na Mesorregião do Agreste e no Sertão Pernambucano, cujas regiões são as montantes de relevo acentuado, conhecidas como brejo e serras úmidas do Agreste e do Sertão Pernambucano.

4. A zona inapta se determinou em 101 localidades distribuídas nas Mesorregiões do Sertão, Sertão de São Francisco e no Agreste Pernambucano, com algumas "ilhas” apontadas como inaptas, na Mesorregião da Zona da Mata, área Norte e Oeste da Mesorregião Metropolitana de Recife.

\section{LITERATURA CITADA}

Assad, E. D. Sistemas de informações geográficas. Aplicações na agricultura. Assad, E. D.; Sano, E. E. (ed.). Brasília: Embrapa-SPI - Embrapa-CPAC, 1998. 434p.
Cavalcanti, E. P.; Silva, E. D. V. Estimativa da temperatura do ar em função das coordenadas locais. In: Congresso Brasileiro de Meteorologia, 12 e Congresso Latino-Americano de Ibérico de Meteorologia, 2, 1994, Belo Horizonte. Anais..., Belo Horizonte: Sociedade Brasileira de Meteorologia, 1994. p.154-157.

Cavalcanti, E. P.; Silva, V. P. R.; Sousa, F. A. S. Programa computacional para estimativa da temperatura do ar. Revista Brasileira de Engenharia Agrícola e Ambiental, v.10, n.1, p.140-147, 2006.

DRA - Diretoria de Rotas Aéreas, Tabelas climatológicas. Rio de Janeiro: Ministério da Aeronáutica. v.1. 1967.

Ellis, J. E.; Valença, A. S. Desvio padrão da temperatura média mensal no Brasil. Brasília: Instituto Nacional de Meteorologia, 1982. p.1-75. Boletim Técnico 22.

Ferreira, A.; Souza Filho, I. C.; Albuquerque; S. C.; Brasil, H. S. Diagnóstico da floricultura em Pernambuco. In: Floricultura em Pernambuco. Recife: SEBRAE, 2002. 82p. Série Agronegócio.

Hagiladi, A.; Umiel, N.; Yang X. H.. Curcuma alismatifolia II. Effects of temperature and daylenght on the development of flowers and propagules. Acta Horticulturae, Herzliya, Israel, n.430, p.755-761, 1997. In: Sarmiento, M. J., Kuehny, J. S., (ed.) Growth and development. Responses of Ornamental Gingers to Photoperiod. Hortechnology, v.14 n.1. p.78-82. 2004.

Junqueira, A. H.; Peetz, M. S. Os pólos da produção de flores e plantas ornamentais do Brasil: Uma análise do potencial exportador. Revista Brasileira de Horticultura Ornamental, v.8, n.1/ 2, p.25-47, 2002.

Lamas, A. M. Floricultura tropical: Técnicas de cultivo. Recife: SEBRAE/PE, 2002. 87p.

Reis, A. C. de S. Zoneamento agroclimático para a cafeicultura em Pernambuco. Boletim Técnico do Instituto de Pesquisa Agronômica, v.52. p.1-26, 1972.

SUDENE - Superintendência do Desenvolvimento do Nordeste. Normais Climatológicas da Área da SUDENE. Convênio com o Serviço de Meteorologia, Recife: SUDENE, 1963.

SUDENE - Superintendência do Desenvolvimento do Nordeste. Dados pluviométricos mensais do Nordeste. Recife: SUDENE, 1990 Série Pluviometria 1 a 10.

Thornthwaite, C. W.; Mather, J. R. The water balance. Publication in climatology, Centerton: Laboratory of Climatology, v.8, n.1, 1955. 104p.

Varejão-Silva, M. A. ; Cezar-Barros, A. H. Zoneamento de aptidão climática do estado de Pernambuco para três distintos cenários pluviométricos. Recife: COTEC/Data Agros - Informática \& Informações Agrícolas/Secretaria de Produção Rural e Reforma Agrária de Pernambuco. 2001. 38p. Relatório Técnico

Zhang, J. X.; Huang. J. P.; Lin, L. M. A new favorite in flower markets: Cultivation technique and regulation of flowering of Curcuma alismatifolia. Taiwan Flower Industry, v.92, n.3, p.36-40, 1995. In: Sarmiento, M. J., Kuehny, J. S., Growth and development. Responses of Ornamental Gingers to Photoperiod. Hortechnology. v.14 n.1. p.78-82. 2004. 